\title{
Validação do National College Health Risk Behavior Survey para utilização com universitários brasileiros
}

\author{
Validation of National College Health Risk Behavior Survey \\ to be use with Brazilian college students
}

Carolina da Franca ${ }^{1}$

Viviane Colares ${ }^{1}$
${ }^{1}$ Departamento de Odontologia Preventiva e Social, Faculdadede Odontologia, Universidade de Pernambuco. Av. Gal. N ewton Cavalcanti 1650, Centro. 54753-220

Camaragibe PE. carolinafbandeira@ yahoo.com.br
Abstract The objective of this article is to translate, to adapt and to validate the $\mathrm{N}$ ational College Health Risk Behavior Survey to apply at Brazilian college students. 208 college students from the Federal University of Pernambuco (UFPE) and University of Pernambuco (UPE) participated in the study. The validation was carried through in five stages: (1) translation; (2) retrotranslation; (3) correction and semantic adaptation (cultural adaptation); (4) face validation; (5) test-retest. Adaptations were done to deal with any semantic disagreements found between translation and retrotranslation. After face validation, the questionnaire was reduced from 96 to 52 questions. From the 11 items analyzed, themajority presented good and perfect Kappa: security and violence (Kap$\mathrm{pa}=0.89)$; suicide $(\mathrm{Kappa}=1.00)$; use of the tobacco (Kappa $=0.90)$; drinking consumption (Kap$\mathrm{pa}=0.78)$; cocaine and other drugs consumption (Kappa $=0.70)$; sexual behavior (Kappa $=0,88)$ and corporal weight (Kappa $=0.89)$. Only the item about feeding presented weak Inter-examiner Kappa (Kappa $=0.26)$ and the topic on health information presented moderateKappa (Kappa $=0.56$ ). The average Kappa for all items was good (0.76). The instrument may be considered validated in the Portuguese language in Brazil with acceptable reproducibility.

Key words H ealth behavior, Cross-cultural comparison, Reproducibility of results, $\mathrm{Q}$ uestionnaires
Resumo 0 objetivo desteestudo étraduzir, adaptar evalidar o National College Health Risk Behavior Survey para utilização com universitários brasileiros. Participaram do estudo 208 universitários da UFPE e UPE. A validação foi realizada em cinco etapas: (1) tradução; (2) retrotradução; (3) correção e adaptação semântica (transculturalização); (4) validação de face; (5) teste e reteste. A tradução e retrotradução não apresentaram muitas discordâncias, tendo sido feitas as adaptações necessárias. A pós a validação de face, o questionário foi reduzido de 96 para 52 questões. A reprodutibilidade foi avaliada através do teste de Kappa. D os onze tópicos analisados, a mai oria apresentou Kappa bom a perfeito: segurança e violência (Kappa $=0,89)$; suicídio (Kappa $=1,00)$; uso do tabaco (Kappa $=0,90)$; bebida alcoólica (Kappa $=0,78)$; consumo de cocaína e outras drogas (Kappa $=0,70)$; comportamento sexual $(K a p p a=0,88)$ e peso corporal (Kappa $=0,89)$. A penaso tópico sobrealimentação apresentou Kappa fraco (Kappa $=0,26)$ e o tópico sobre informações em saúde apresentou Kappa moderado (Kappa=0,56). 0 Kappa médio para todos os tópicos foi 0,76 , classificado como bom. A versão reduzida do instrumento pode ser considerada validada na língua portuguesa com nível de reprodutibilidade aceitável.

Palavras-chave Conduta de saúde, Comparação transcultural, Reprodutibilidade dos testes, Questionários 
Introdução

Conduta de saúde é um tema que tem sido bastante debatido em todo o mundo, principalmente por sua relação com as doenças crônicas. A adoção de condutas saudáveis pode prevenir ou retardar doenças crônicas que são responsáveis por $60 \%$ de todas as mortes no mundo ${ }^{1}$.

$\mathrm{O}$ questionário $\mathrm{N}$ ational College $\mathrm{H}$ ealth Risk Behavior Survey (NCHRBS), desenvolvido pelo Centersfor Disease Control and Prevention (CDC) faz parte do projeto americano $\mathrm{H}$ ealthy People 2010, que visa monitorar comportamentos de risco para a saúde de adolescentes e jovens dos Estados Unidos. Este questionário proporciona uma visão geral das condutas de saúde através da abordagem de temas centrais como uso do tabaco, comportamento alimentar, prática de atividade física, uso do álcool e outras drogas, comportamento sexual e comportamentos relacionados à violência².

Muitos jovens, ao se engajarem na universidade, passam a adotar comportamentos de risco que podem afetar a saúde dos mesmos em longo prazo. 0 monitoramento de condutas de saúde permite que as políticas de prevenção e promoção sejam instituídas de manei ra direcionada. Além do monitoramento, éfundamental a educação em saúde para que os indivíduos sejam ativos e consequentemente incorporem práticas saudáveis de vida tendo maior responsabilidade sobre a própria saúde’3.

0 ambiente universitário geralmente é o lugar onde os jovens têm pela primeira vez total independência da supervisão dos pais. N esta fase, os indivíduos real mente tornam-se responsáveis por todos os seus comportamentos e tendem a questionar suas crenças e valores. É nesse período que os estudantes podem mudar seu estilo de vida e passar a adotar condutas positivas ou negativas para a saúde'.

Quanto mais cedo os indivíduos adotarem condutas de saúde positivas, maiores serão as chances dessas condutas persistirem ao longo da vida adulta. Segundo Tamin et al. ${ }^{4}$, os comportamentos formados na segunda década de vida têm consequências duradouras para o indivíduo e para a saúde pública em geral.

Os universitários da área de saúde serão os futuros profissionais que deverão atuar como educadores e motivadores para a adoção de condutas saudáveis pela população em geral.

No Brasil, ainda não há nenhum instrumento validado que aborde as condutas de saúde entrejovens universitários. O NCHRBSéum ins- trumento que aborda os principais temas desaúde, fornecendo um panorama das atitudes dos indivíduos em relação a sua saúde.

Assim, este trabal ho teve como objetivo realizar a tradução, a adaptação cultural e a avaliação da reprodutibilidade da versão reduzi da em português do NCH RBS para aplicação com universitários brasileiros. Desta forma, este instrumento poderá ser mais uma ferramenta disponível para utilização em pesquisas a fim de monitorar as atitudes relacionadas à saúde de jovens universitários.

\section{Métodos}

O NCHRB é um instrumento composto por 96 questões, distribuídas em grupos de perguntas, de acordo com os temas abordados: dados sociodemográficos (catorze questões), segurança e violência (quinzequestões), suicídio (quatro questões), tabaco (oito questões), bebida alcoólica (três questões), maconha (três questões), cocaína e outras drogas (dez questões), comportamento sexual (quinze questões), peso corporal (oito questões), alimentação (sete questões), atividade física (seis questões) e informação sobre saúde (três questões).

0 processo de validação foi realizado em cinco etapas: (1) tradução; (2) retrotradução; (3) correção e adaptação semântica (transculturalização); (4) validação de face; (5) aplicação do instrumento com teste e reteste para avaliar a confiabilidadeereprodutibilidadedo instrumento (Figura 1).

Participaram das etapas do processo de validação 208 estudantes universitários na faixa etária de dezoito a 28 anos, de ambos os sexos, das universidades públicas de Pernambuco: UFPE e UPE.

$\mathrm{O}$ instrumento $\mathrm{NCH}$ RBS foi submetido àtradução reversa para obtenção da equivalência semântica, ou seja, manter o significado de cada item do instrumento após a tradução para outra língua diferente da original ${ }^{5}$. Esse processo consistiu na tradução do instrumento original em inglês para o português (primeira etapa) e retrotradução da versão em português para o inglês (segunda etapa). Essas duas etapas foram realizadas por dois profissionais com proficiência nas línguas inglesa e portuguesa. A língua materna de um era a portuguesa e a do outro era a inglesa. Não foi mantido qualquer contacto entre os profissionais durante a tradução, obtendo-se assim dois textos independentes. Os tradutores 


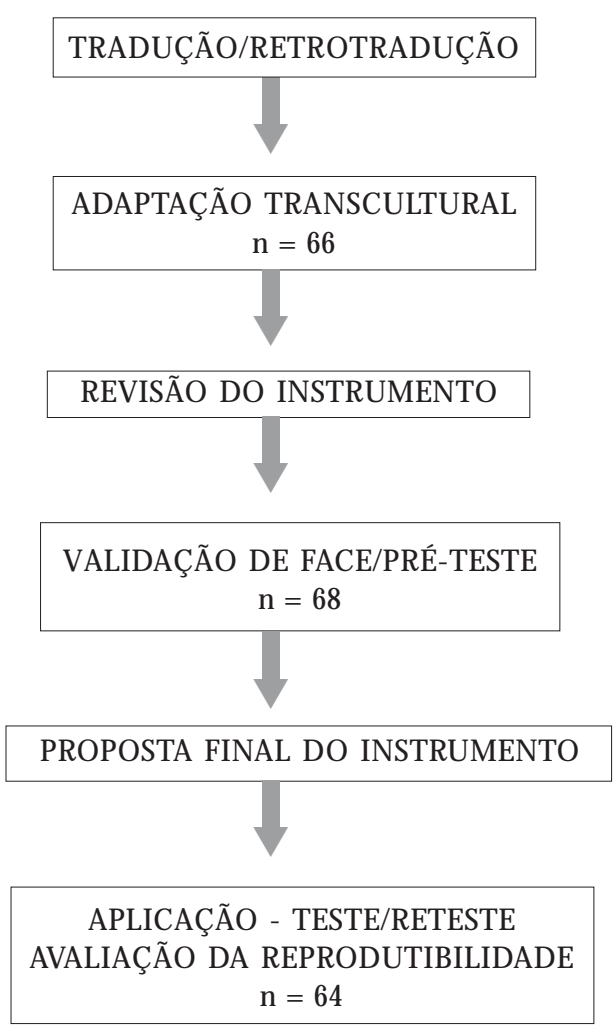

Figura 1. Fluxograma da validação do instrumento.

foram orientados a traduzir atentamente as interpretações divergentes de itens ambíguos do texto. Foi realizada revisão pelos autores deste estudo e pelos tradutores do instrumento para ajustes da tradução e adaptação cultural (terceira etapa).

A validação de face (quarta etapa) visou adequar o instrumento ao nível deentendimento dos universitários. Essa fase foi realizada em dois momentos, que contribuíram para redução no número de questões.

A objetividade é um indicador de reprodutibilidade que se refere à administração do instrumento. Um testeobjetivo éaquele que sofrepouca interferência do aplicador 6 . Assim, visando testar a objetividade do instrumento em questão, foi realizada a aplicação do questionário por dois examinadores diferentes.

Para o teste da reprodutibilidade, o questionário foi aplicado ereaplicado (quinta etapa) no mesmo grupo de indivíduos apósum período de dez a dezenove dias, verificando, dessa forma, a consistência das respostas obtidas. Este interva- lo de tempo pode ser considerado, como sugere Picon et al. ${ }^{7}$, nem tão curto que permita o viés de aferição por lembrança das respostas do questionário e nem tão longo que leve a modificações nas respostas devido a alterações nas condutas de saúde.

Para análise dos dados, foram obtidas distribuições absolutas e percentuais de cada questão. Com o objetivo de verificar o grau de coincidência intraexaminadores e interexaminadores, foram obtidos os escores de coincidência deK appa para cada questão, incluindo a obtenção de um intervalo de confiança para o Kappa populacional. Nas questões em que os itens não estavam distribuídos em escala ordinal, foi aplicado o Kappa ponderado.

A escal a utilizada para os escores de Kappa foi a de Landis e Koch, citados por Pereira8: 0,20 (pobre); 0,21-0,40 (fraca); 0,41-0,60 (moderada); 0,610,80 (boa); 0,81-0,99 (ótima); 1,00 (perfeita).

Os dados foram digitados através na planilha Excel e o software utilizado para a obtenção dos cál cul os estatísticos foi o SAS (Statistical Analysis System) na versão 8.0 para microcomputador.

\section{Considerações éticas}

Em todas as fases, o termo de consentimento foi assinado pelos participantes voluntariamente, sendo garantido 0 anonimato e 0 absoluto sigilo. 0 projeto de pesquisa foi aprovado pelo Comitê de Ética da Universidade de Pernambuco.

\section{Resultados}

Participaram da validação 208 estudantes universitários subdivididos nas seguintes etapas: 66 estudantes na realização da adaptação transcultural, 68 estudantes na segunda validação de face e pré testee 74 estudantes na avaliação da reprodutibilidade. Durante a avaliação da reprodutibilidade, houve uma perda de dez estudantes que não foram localizados eseis estudantes que identificaram o questionário no reteste de maneira diferente do testeinicial.

\section{Tradução e retrotradução}

Não se observou discordâncias importantes no significado entre a versão original e a versão resultante da tradução inversa. As diferenças encontradas apresentavam significados equivalentes ou sinônimos. 


\section{Adaptação transcultural}

No processo de adaptação, algumas questões foram modificadas para facilitar 0 entendimento dos estudantes, optando-se pela utilização de sinônimos que se adaptavam melhor à linguagem dos universitários.

\section{Validação de face e pré-teste}

A validação defaceocorreu em dois momentos; no primeiro, foi utilizada a versão completa do questionário e, no segundo momento, foi utilizada a versão reduzida.

A aplicação da versão completa foi realizada em sala de aula e teve duração entre 20 e $25 \mathrm{mi}$ nutos. A duração de aplicação foi considerada longa e exaustiva. Para maior objetividade do instrumento, 37 questões que apresentaram baixo índice de respostas pelos estudantes foram retiradas do instrumento.

Assim elaborou-se uma versão reduzida, mantendo-se, porém, todos os temas abordados na versão original. A primeira parte do instrumento, que trata do perfil sociodemográfico, foi reduzida de catorze para seis questões.

0 tópico sobre alimentação teve o número de questões reduzido de sete para quatro. Porém, nesse tópico, as perguntas foram agrupadas, como por exemplo, as questões 81 e 82 , pois a primeira questão se referia ao número de vezes que o universitário havia comido frutas e a segunda, ao número de vezes que o universitário havia tomado suco de frutas. Assim, questionou-se em uma única pergunta quantas vezes 0 universitário havia comido frutas ou tomado suco de frutas.

0 tópico referenteao tema maconhafoi mantido com três questões, assim como aquele sobre peso corporal foi mantido com oito questões.

A pós as reduções e 0 acréscimo de duas perguntas, o questionário passou de 96 para 52 questões. Em relação às duas questões acrescidas, uma, a respeito do uso do cinto de segurança em automóveis, foi dividida em duas para que fosse discriminado o uso do cinto no banco da frente ou no banco de trás do carro. A outra questão acrescentada foi na secção sociodemográfica sobre a percepção que os estudantes têm da sua saúde geral.

Avaliação da consistência interna

Após a redução do questionário, foi realizada a avaliação da consistência interna. 0 alfa de
Cronbach mostrou uma consistência interna aceitável $(0,667)$.

\section{Avaliação da reprodutibilidade}

Durante esta última fase, o instrumento foi aplicado duas vezes. No teste inicial, participaram 74 estudantes que identificaram seus questionários com as iniciais de seu nome para posterior comparação no reteste. Após um período entre dez edezenove dias, os mesmos participantes responderam novamente o questionário para verificação da reprodutibilidadee confiabilidade dos resultados.

No reteste, 64 estudantes participaram, porém seis colocaram iniciais diferentes daquelas do teste e por isso foram excluídos, restando 58 participantes, ou seja, 78,3\% dos estudantesidentificaram o questionário da mesma forma na primeira e segunda vez.

Em relação à reprodutibilidade e objetividade, os resultados estatísticos (Tabelas 1 e 2) mostraram que a maioria dos blocos de perguntas apresentou um grau de coincidência intraexaminador e interexaminador com Kappa bom e ótimo. Isso mostra que o teste para reprodutibilidade do instrumento foi aceitável para a maioria dos onzetemas abordados no questionário. Apenas dois blocos de perguntas na análise interexaminadores apresentaram o Kappa fraco e moderado, que abordavam os temas alimentação e informações em saúde, respectivamente. $\mathrm{N}$ a análise intraexaminadores, estes dois tópicos apresentaram o Kappa moderado e bom, respectivamente. Ainda nesta segunda análise, o tópico sobre atividade física mostrou um Kappa moderado. É provável que esse Kappa baixo (fraco e moderado) tenha ocorrido nos tópicos sobrealimentação e atividade física devido às questões desses blocos se referirem à alimentação do dia anterior e prática de atividade física nos últimos sete dias. Dessa forma, é possível que os estudantes no intervalo de dez a dezenove dias tenham se alimentado e se exercitado de maneira diferente por razões independentes desse estudo e por isso não venha a comprometer o resultado geral da validação.

\section{Discussão}

Vários estudos têm sido realizados no mundo sobre condutas de saúde e o questionário desenvolvido pelo CDC tem sido utilizado em pesquisas desenvolvidas com jovens americanos ${ }^{9-12}$, as- 
Tabela 1. Avaliação do grau de coincidência intraexaminador por bloco de perguntas.

\begin{tabular}{|c|c|c|c|}
\hline \multirow[b]{2}{*}{ Bloco de perguntas } & \multicolumn{3}{|c|}{ Concordância observada } \\
\hline & $\mathrm{n}$ & $\%$ & Kappa IC (95\%) \\
\hline Segurança e violência & 147 & 84,5 & $0,85(0,79 \text { a } 0,92)^{(*)}$ \\
\hline . Suicídio & 49 & 98,0 & $0,96(0,88$ a 1,00$)$ \\
\hline . Uso do tabaco & 71 & 97,3 & $0,95(0,88$ a 1,00$)$ \\
\hline . Consumo de bebida alcoólica & 32 & 65,3 & $0,77(0,67 \text { a } 0,87)^{(*)}$ \\
\hline . Consumo de maconha & 73 & 97,3 & $0,77(0,49$ a 1,00$)$ \\
\hline . Consumo de cocaína e outras drogas & 169 & 96,6 & $0,76(0,59 \text { a } 0,93)^{(*)}$ \\
\hline . Comportamento sexual & 325 & 92,9 & $0,91(0,87 \text { a } 0,95)^{(*)}$ \\
\hline . Peso corporal & 112 & 90,3 & $0,89(0,83 \text { a } 0,95)^{(*)}$ \\
\hline . Alimentação & 51 & 51,0 & $0,42(0,29 \text { a } 0,56)^{(*)}$ \\
\hline - Atividade física & 10 & 40,0 & $0,55(0,31 \text { a } 0,79)^{(*)}$ \\
\hline . Informação em saúde & 218 & 87,2 & $0,74(0,66$ a 0,83$)$ \\
\hline . Total das perguntas de todos os blocos & 1256 & 86,8 & $0,80(0,77$ a 0,82$)$ \\
\hline
\end{tabular}

${ }^{(*)}$ Kappa ponderado.

Tabela 2. Avaliação do grau de coincidência interexaminadores por bloco de perguntas.

\begin{tabular}{|c|c|c|c|}
\hline \multirow[b]{2}{*}{ Bloco de perguntas } & \multicolumn{3}{|c|}{ Concordância observada } \\
\hline & $\mathrm{n}$ & $\%$ & Kappa IC (95\%) \\
\hline Segurança e violência & 212 & 86,5 & $0,89(0,85 \text { a } 0,93)^{(*)}$ \\
\hline Suicídio & 70 & 100,0 & $1,00(1,00$ a 1,00$)$ \\
\hline . Uso do tabaco & 99 & 95,2 & $0,90(0,83$ a 0,98$)$ \\
\hline . Consumo de bebida alcoólica & 47 & 67,1 & $0,78(0,69 \text { a } 0,88)^{(*)}$ \\
\hline . Consumo de maconha & 104 & 99,0 & $(* *)$ \\
\hline - Consumo de cocaína e outras drogas & 240 & 97,9 & $0,70(0,39 \text { a } 1,00)^{(*)}$ \\
\hline . Comportamento sexual & 411 & 91,7 & $0,88(0,83 \text { a } 0,93)^{(*)}$ \\
\hline . Peso corporal & 161 & 92,0 & $0,89(0,83 \text { a } 0,95)^{(*)}$ \\
\hline Alimentação & 55 & 39,6 & $0,26(0,14 \text { a } 0,39)^{(*)}$ \\
\hline - Atividade física & 16 & 45,7 & $(* *)$ \\
\hline . Informação em saúde & 275 & 78,6 & $0,56(0,47$ a 0,65$)$ \\
\hline . Total das perguntas de todos os blocos & 1.690 & 85,1 & $0,76(0,73$ a 0,78$)$ \\
\hline
\end{tabular}

(*) Kappa ponderado.

(**) Não foi determinado devido à diferença no número de categorias entre as duas avaliações.

sim como os resultados encontrados pelo CDC em 1995 têm sido utilizados como importante referência para comparações posteriores.

No Brasil, apenas o questionário desenvolvido pelo CDC voltado para adolescentes (Youth Risk Behavior Survey) foi traduzido e adaptado à realidade brasileira por Carlini-Cotrim et al. ${ }^{13}$.

A validação desse questionário seguiu metodologia semel hante a outros estudos de validação $0^{7,14-16}$.
Com base nos resultados, é possível afirmar que o instrumento NCHRBS é objetivo e reprodutível com níveis aceitáveis do Kappa para todos os blocos de perguntas com exceção daquelas a respeito da alimentação eda atividadefísica. Para esses dois tópicos, não foi possível uma boa análise da reproduti bilidade em função da variação normal de um dia para o outro na alimentação e atividade física dos indivíduos de modo geral. Os resultados demonstraram que a ativi- 
dade física dos estudantes não seguiu um padrão regular semanal. No caso da alimentação, o questionário foi referente ao recordatório do dia anterior, o que dificulta uma avaliação semeIhante devido a uma variação esperada na dieta diária dos estudantes, especialmente após um intervalo de dez a dezenove dias.

A pesar dos resultados positivos na validação do questionário, o processo apresentou al gumas limitações. Para se obter veracidade nas respostas fornecidas pelos estudantes, foi proporcionada privacidade e segurança no sigilo. No entanto, condutas de saúde como comportamento sexual e abuso de drogas podem ter constrangido ou intimidado os estudantes. Outra limitação foi decorrente da extensão do questionário, que conteve em sua versão final 52 perguntas, 0 quefez al guns estudantes recusarem-sea participar, e outros o fizeram rapidamente, aparentemente sem a concentração necessária. A necessi- dade da identificação dos participantes no questionário para a realização do reteste, assim como aidentificação no termo de consentimento livree esclarecido, também atuaram como inibidores no processo de coleta de dados. Para minimizar essa limitação, solicitou-se que os próprios jovens colocassem o questionário eo consentimento em envelopes distintos, proporcionando maior sigilo. A pesar disso, alguns universitários responderam o questionário, porém, não assinaram 0 termo de consentimento; e outros identificaram o questionário deformaquenão foi possível identificar o reteste. No entanto, essas limitações são esperadas em pesquisas que avaliam comportamento envolvendo jovens, sendo possível minimizar as interferências de forma que não comprometa os resultados do estudo.

Dessa forma, o questionário NCHRBS foi traduzido, adaptado transculturalmente e a reprodutibilidade foi considerada satisfatória.

\section{Colaboradores}

C da Franca e V Colares trabalharam na pesquisa, análisee interpretação dos dados e redação do artigo.

\section{Agradecimentos}

Aos estudantes da UPE e da UFPE que participaram voluntariamente deste estudo, respondendo o questionário em todo o processo de validação. 


\section{Referências}

1. Organização M undial de Saúde. Prevenção de Doenças Crônicas - um investimento vital. [site da Internet] [acessado 2005 nov 03]. Disponível em: http:/ /www.opas.org.br/sistema/fotos/DCNT_OM S.pdf

2. Centers for Disease Control and Prevention. Youth Risk Behavior Surveillance: National College Health Risk Behavior Survey - United States, 1995. M M W R [periódico na Internet]. 1997 [acessado 2005 jul 10];46(14):[ cerca de 64 p.]. Disponível em: http:/ /www.cdc.gov/mmwr/PDF/ss/ss4606.pdf

3. Rozmus CL, Evans R, Wysochansky M, Mixon D. An analysis of health promotion and risk behaviors of freshman college students in a rural southern setting. J Pediatr N urs 2005; 20(1):25-33.

4. Tamim H, Terro A, Kassem H, Ghazi A, Khamis TA, Hay MMA, Musharrafieh U. Tobacco use by university students, Lebanon, 2001. Addiction 2003: 98:933-939.

5. Herdman M, Fox-Rushby J, Badia X. A model of equivalence in the cultural adaptation of $H R Q O L$ instruments: the universalist approach. Qual Life Res 1998; 7:323-335.

6. Barros M VG, Nahas MV. Reprodutibilidade (testereteste) do Questionário Internacional de Atividades Físicas (QIAF - Versão 6): um estudo piloto com adultos no Brasil. Rev Bras Ciênc M ov 2000; 8:23-26.

7. Picon P, Gauer GJC, Hirakata VN, Haggsträm LM, Beidel DC, Turner SM, Manfro GG. Reliability of the Social Phobia and Anxiety Inventory (SPAI) Portuguese version in a heterogeneous sample of Brazilian university students. Rev Bras Psiquiatr. 2005; 27(2):124-130.

8. Pereira M G. Epidemiologia: teoria e prática. Rio de Janeiro: Guanabara Koogan; 2005.

9. Barrios LC, Sherry AE, Simon TR, Brener ND. Suicide ideation among US college students. J. Am. Coll. Health 2000; 48:229-233.
10. Jones SE, Oeltmann J, Wilson TW, Brener ND, Hill CV. Binge drinking among undergraduate college students in the United States: implications for other substance use. Journal of American College Health 2001; 50:33-38.

11. Lowry R, Galuska DA, Fulton JE, Wechsler H, Kann L, Collins JL. Physical activity, food choice and weight management goals and practices among US college students. Am J Prev M ed 2000; 18(1):18-27.

12. O'M alley PM, Johnston LD. Epidemiology of alcohol and other drug use among American College Students. J Stud Alcohol Suppl 2002; (Suppl.) 14:23-39.

13. Carlini-Cotrim B, Gazal-Carvalho C, Gouveia N. Comportamentos de saúde entre jovens estudantes das redes pública e privada da área metropolitana do Estado de São Paulo. Rev. Saude Publica 2000; 34(6):636-645.

14. Avanci JQ, Assis SG, Santos NS, Oliveira RVC. Escala de violência psicológica contra adolescentes. Rev. Saude Publica 2005; 39(5):702-708.

15. Grassi-Oliveira R, Stein LM, Pezzi JC. Tradução e validação de conteúdo da versão em português do Chilhood Trauma Questionnaire. Rev. Saude Publica 2006; 40(2):249-255.

16. Griep RH, Chor D, Faerstein E, Lopes C. Confiabilidade teste-reteste de aspectos da rede social no Estudo Pró-Saúde. Rev. SaudePublica 2003; 37(3):379385.

Artigo apresentado em 22/10/2007

Aprovado em 31/03/2008 